coursing septic bullet wound from the outer to the inner side of the thigh 7 inches above the knee-joint. Operation five weeks after infliction. A median anterior incision 8 inches in length was made. The bone was defined, and three fragments, after much difficulty. owing to rigidity of the muscles, were manipulated into place. The middle flake accidentally was now detached in moving a retractor and fell on the floor. Two short plates were then placed on (1) the inner side of the shaft of the femur; and (2) the outer aspect of the inner side of the compact bone of femoral shaft-i.e., in the medullary cavity. (Fig. 4.) The incision healed per primam, but the bullet track still discharges, a sinus 8 inches long still being present after six weeks.

There is good union with much callus. The plates in this case may yet need removal, but in the last few days the sinus has become somewhat smaller, and I shall continue conservative treatment for 14 days more before removal of the plates, if then necessary. The position is as good as before the fracture.

CASE 5.-Private ; comminuted fracture of lower third of tibia. The fracture was exposed and the fragments were sewn in position with silkworm gut. Union took place in four weeks in perfect position.

CASE 6.-Private; Alpine Chasseurs; 6 inches blown away from the middle of the shaft of the femur by a bullet at short range. Six weeks after infliction, the wounds being fairly clean, 8 inches of the fibula were resected from the same leg and placed in the gap by forcing it into the medullary cavity of the upper and lower fragments. A good deal of suppuration took place after the operation, but the fibular fragment was not extruded. Eventually the wounds healed and there was 2 inches' shortening of that limb. The patient was discharged from hospital in plaster-of-Paris. I was quite unable to follow this case after he had been sent to his (French) depôt.

Conclusions.-1. Time is saved by the above method if early fixation of bone is undertaken, one or at the most two operations are necessary, and union generally takes place as in a simple fracture, though somewhat delayed by open wounds. 2. Almost complete absence of pain. 3. Callus formation in spite of suppuration; in some cases it even seems to be stimulated to exuberance by it. 4. Foreign bodies-i.e., plates and shrapnel-even when septic, can be rendered sterile and tolerated by the tissues. 5. Position is almost as good as a simple fracture treated by plating. 6. Where a large wound in the soft parts is necessary for cleaning and drainage, rationally the bone fragments should be manipulated into position. 7. Dressing is facilitated by a "fixed" fracture, even if this fixation be but partial. 8. Function is better than by other methods.

My thanks are due to Lieutenant-Colonel Maurice, R.A.M.C. (T.F.), for valuable advice and permission to deal with the above cases (except Case 6), and to Captain Foster, R.A.M.C. (T.F.), for the originals of the radiographs.

UNiversity of London : UnIVERsity College. -The following elections to scholarships have been made :Bucknill scholarship (135 guineas), J. P. Padshah. Medical entrance exhibitions (55 guineas each). H. L. Heimann and A. B. Saunders. Epsom free medical scholarship, D. C. Corry.

Memorial to the LATE Mr. EdGar JoHN Donbavand, L.R.C.P., M.R.C.S.-A stained-glass window, erected to the memory of the late Mr. E. J. Donbavand, was unveiled last week at Arlesey Church, Bedfordshire. The window is a good specimen of fourteenth century style and contains a representation of St. Luke as the central piece. Mr. Donbavand, who died at Plymstock (Devon) in 1914, was formerly in practice at Arlesey.

\section{INTESTINAL DRAINAGE IN SEPTIC PERITONITIS.}

\author{
BY ARTHUR J. NYULASY, L.R.C.P.LOND., \\ M.R.C.S., \\ GYNACOLOGIST TO THE PERTH HOSPITAL, AUSTRAIIA.
}

THE examination after death of a large series of cases of septic peritonitis reveals two fairly distinct pathological types: (1) that in which there is abundance of fluid free in the abdomen but little actual peritonitis; and (2) that in which there is little or no free fluid but extensive peritonitis. In conformity with custom the term "septic peritonitis" is here employed to refer to either type, but it is to be clearly understood that this expression must be regarded as clinical and provisional rather than as the name of a definite pathological entity sufficient in itself to account for death. Although pathologically the two types differ so markedly, they agree in having intestinal paralysis as a great clinical feature. In the one type this apparently results from infective inflammation of the intestinal peritoneum; in the other it seems to be due to toxic fluid free in the abdomen acting on the intestinal nerves, and inhibiting peristalsis. Be the explanation of the acute stasis what it may, the deterioration and death of the patient is unquestionably due-in many cases largely, and in others almost entirely-to resulting intestinal toxæmia. The rational treatment of this toxæmia is the removal of unabsorbed intestinal toxin and the prevention of its further production. Both these ends may be secured by intestinal drainageprovided that the peritoneal cavity is not charged with excessive poison and that the focal factor is eliminated.

As all the cases described in this article followed on abdominal operations, it will be well to indicate the main points in the diagnosis of post-operative septic peritonitis. Progressive deterioration is the great outstanding feature, the downward process usually beginning 12 to 24 hours after operation. The patient rapidly loses strength, and finally looks so very ill as to suggest the work of some virulent poison. Both pulse and respiration rate quickly reach a high grade, while the blood pressure markedly falls. Abdominal pain is not a constant symptom, the patient perhaps making no complaint whatever, merely being very restless. When the pain is due to actual inflammation there is coincident marked tenderness to pressure, while when due to distension it is liable to be more colicky. Although, as a rule, the abdomen is distended, this sign may be absent, and some of the worst cases of septic peritonitis may show a scaphoid abdomen. Abdominal movement is diminished or practically lost. Vomiting is in the majority of instances a striking feature and may actually become fæcal, but in some cases it is comparatively slight, the diagnosis then depending on the rapid general deterioration. When the deterioration is fully established the passage of gas and fæces usually ceases, although enemata may bring away both fæces and flatus. In other instances there may be actual diarrhoea. Here, again, the diagnosis hinges largely on the rapid general deterioration.

In the Perth Hospital, Australia, my last six cases of post-operative septic peritonitis have been treated by intestinal drainage, and all the cases have recovered, a result which $I$ am convinced could not have been achieved by any other known 
method of treatment. To make my attitude more clear, the following summary of the treatment may be stated. 1. If the focal factor has been eliminated, free toxic fluid mopped away, septic necrotic areas drained, the Fowler position employed, saline freely administered, and early purgation given a trial, but the patient nevertheless continues to deteriorate from toxæmia due to paralytic ileus, then intestinal drainage should be instituted. 2. If, at the primary operation, drainage of the septic focus in the abdomen has not been employed and the patient deteriorates, a drainagetube may now be introduced; if the patient does not then rapidly improve, but continues to go down hill, intestinal drainage may now be instituted under local anæsthesia. 3. If, when the case is first seen, the evidence of toxæmia from paralytic ileus is positive, and especially if lividity is obvious, then intestinal drainage should form part of the primary operation.

CASE 1. Appendicectomy ; localised (?) intestinal paralysis ; cacostomy.-A multipara, aged 38 years. Operation on May 29th, 1912: Curettage, excision of cyst of right ovary, looping left ovarian ligament, hysteropexy, and appendicectomy for subacute appendicitis, the appendix being removed by my assistant. Next day there was marked pain and tenderness over the cæcum and ascenaing colon. The following day the patient was collapsed (temperature $97^{\circ} \mathrm{F}$. pulse 140), but refused operation till a week later, when, after slight improvement from the collapse, her condition had become very grave, the symptoms (severe pain and tenderness, slight vomiting, fluctuating temperature, pulse 120 to 140 , but fæces and flatus passing) gradually becoming worse. She had a bad colour, and looked very ill and depressed.

On June 7th through an oblique incision the cæcum and colon were found to be adherent to the abdominal wall, and intensely inflamed, thick, stiff, and friable. A rubber tube was purse-strung into the cæcum. The patient thence onward slowly improved. At the end of a week, on giving an enema, the fluid ca me out not only through the cæcostomy opening, but also through a sinus which had formed in the lower end of the suprapubic incision. Both openings closed in a few weeks. In this case there had apparently been some fault in the technique of removal of the appendix, leading fortunately to only a localised peritonitis.

Case 2. Paralytic ileus with free diarrheea and definite peritonitis ; enterostomy. - A multipara, aged 39 years. Operation on March 5th, 1913: Curettage, salpingectomy and appendicectomy (rather recent sa'pingitis and appendicitis), hysteropexy. A small hard lump at the uterine end of the right round ligament was found to contain about half a drachm of yellowish pus, the cavity being cleansed and swabbed out with pure carbolic and alcohol.

A few days later an oblique incision was made over the cæcum and pus was found to well up from the locality of the uterus; a rubber drainage-tube was inserted. On March 11th the report was that the patient had been rather drowsy and indifferent since the second operation, while free diarrhca had continued. She now looked markedly drawn and weary, her colour was very bat, the tongue was dry and coated, the temperature was $102^{\circ} \mathrm{F}$., the pulse-rate 140, and respirations 40. Abdominal distension had progressively increased, and was now so pronounced as to suggest that the intestine might possibly burst. It seemed obvious that unless relief was promptly given the patient must die. Enterostomy was at once carried out through the upper end of the suprapubic incision and proved trying on account of the wound being infiltrated with pus and all the tissues markedly friable. There was obvious peritonitis up to and past the upper end of the wound (which reached the umbilicus), blood-stained fluid being present in the abdomen, the small intestines presenting purplish, and the coils so adherent that the selected one had to be broken away to bring it up to the wound. The parietal peritoneum was so friable that sutures tore out, and I had to be content to stitch the bowel to the subcutaneous fat. A rubber tube was fixed into the bowel. The blood-stained fluid in the abdomen was only roughly mopped up. In a cuuple of days the abnormally distended abdomen had become flat, but later, when the enterostomy tube came away, the freces escaping on to the skin caused much misery. In about six weeks the patient was discharged with all wounds healed.

CASE 3. Salpingeotomy; paralytio ileus; secondary vaginal drainage and cacostomy. - $\mathrm{A}$ woman, aged 23 years, was admitted with a three months criminal abortion, and the uterus was promptly cleared out. The patient deteriorated, and a couple of days later the pulse was 135 and the respirations 36. She looked pale and distressed, and the tongue was drying.

On March 11th, 1913, operation revealed free bloodstained fluid filling and extending up from the pelvis. After breaking down adhesions, a right tubo-ovarian abscess containing horribly foul pus was found. When this had been dealt with the patient's condition was rery grave, so that I rapidly closed the abdomen. During the next few days the patient was obviously deteriorating, the face being pale and drawn, and the lips and ears darkening, while the pulse was very poor and quick. The bowels were acting with purges and enemata, and there was only. slight vomiting. On the 18th the abdomen was greatly distended and immobile, while there were marked tenderness and thickening in Douglas's pouch, and the patient complained greatly of pelvic pain. There was swelling of the vulva, a sign noted in the preceding case. Cæcostomy was carried out; the cæcum was healthy and turbid free fluid was mopped out from the pelvis through the oblique abdomioal incision. Douglas's pouch was then opened through the vagina and gave exit to stinking, thin sero-pus. The patient did well, the distension and other symptoms rapidly improving.

CaSE 4. Paralytic ileus; secondary abdominal drainage and cacostomy (an unrehearsed clinical experiment).-A multipara, aged 35 years Operation July 22nd, 1914: Densely and extensively adherent pus sacs were excised, the stump of the left broad ligament being so friable that ligatures cut through as if it were cheese; inflamed appendix removed. The patient progressively deteriorated, and about 48 hours after operation was in a very grave state. She looked drawn and anxious, her colour was bad, the tongue was dry, there was much abdominal pain, vomiting was almost continuous, the abdomen was enormously distended, the hands were cold and clammy, and the pulse was a mere thread. The temperature was $97^{\circ} \mathrm{F}$., the pulserate 140 , and the respirations 40 , and in every way the case looked very hopeless.

On opening the abdomen over the cæcum a good deal of watery blood-stained fluid appeared, and was fairly well mopped out. A rubber tube was fixed in the cæcum. The lower end of the median abdominal wound was next opened, and a good deal of blood-stained fluid mopped out of the pelvis, a rubber tube being then inserted. When seen about two hours later the patient had become delirious, and so obstreperous that it had been found necessary to restrain her. I discovered that the cæcostomy tube had been blocked. This was remedied, and the improvement thence onward until next day was so consistent that I felt satisfied that the preliminary failure of the cæcostomy tube had permitted toxin to remain in the bowel and set up delirium. This course of events also suggested the likely progress of the case to a fatal ending if creostomy had not been carried out. Improvement was rapid, and two and a half days after the operation the patient was reading the newspaper.

CASE 5. Salpingectomy; paralytio ileus ; oacostomy.-A multipara, aged 33 years, was admitted with double pyosalpinx, some ounces of foul pus having been passed per rectum a few days earlier.

Operation (July 29th, 1914) revealed a very densely and extensively adherent left tubo-ovarian abscess, containing about 10 ounces of horribly fœtid pus. After salpingooöphorectomy a large dirty necrotic surface was left behind the broad ligament and uterus. A medium-sized right pyosalpinx was also removed. A rubber tube was passed down to Douglas's pouch. Shock was so profound that it was impossible to place the patient in the Fowler position until the next day, when she complained much of "sinking through the bed." Thence onward she deteriorated, and by noon of July 31st it was apparent that a great change for the worse had taken place. The patient looked critically ill ; the face was pale, the lips were livid, and the nose was pinched; 
the pulse-rate was 144 , the respirations 44 , and the temperature $96^{\circ} \mathrm{F}$.; the abdomen was considerably distended, but vomiting was not urgent.

Without altering the position of the patient in bed, and under local anæsthesia, an oblique incision was made over the cæcum, saline being run into the median basilic during operation. Sero-pus was mopped out of the pelvis, and a rubber tube fixed in the cæcum. The wicking was removed from the median drainage-tube, and a little stinking pus sucked out by a syringe. At the close of the operation dissolution seemed imminent, but in spite of the gloomy outlook the patient rallied, and on the fifth day she was taking fish.

CASE 6. Ectopic gestation; paralytic ileus with facal vomiting; ccecostony.-A woman, aged 24 years, was admitted with a history pointing to rupture of an ectopic gestation three weeks earlier. She was very anæmic, the pallor having that “dirty" appearance suggesting sepsis. The pulse-rate was 136 , the temperature $99.6 \mathrm{O}$ F., and the respirations 28 .

At the operation (May 5th, 1915) the abdomen was found to be full of very dark fluid blood and clots. A fœetus, $4 \frac{1}{2}$ inches long with well-developed placenta, was removed, and left salpingectomy carried out. In spite of the employment of many swabs and much saline, it was found impossible thoroughly to cleanse the abdomen of clots, and finally, while the washings were still very dark, I was compelled to close the wound on account of the poor condition of the patient. The next day there was a good deal of vomiting and distansion. On the following day it was reported that both these symptoms had progressively increased during the preceding night, and that fæcal vomiting had set in. At 8 A.M. on this day 20 ounces of fluid had been brought up at one bout. This fluid had a strikingly fæcal odour, and in every way suggested the bowel contents. Vomiting thence onwards was continuous and profuse, 18 ounces being brought up about noon, when I decided to operate. At this time the patient was in an extremely critical condition, and there seemed very little hope of recovery. The pulse was 160 , temperature $96.4{ }^{\circ}$, and respirations 40 ; the abdomen was immobile and enormously disterded, the extremities were cold and tremulous, and the patient looked intensely pale and ill. On opening the abdomen over the cæcum mach dark bloody fluid welled out on to the bed. It seemed to me that this fluid represented the last washings left in the abdomen at the primary operation. No attempt was now made thoroughly to get out this fluid from the abdomen, only the easily accessible surplus being mopped away. A rubber tube was pursestrung into the cæcum and connected with a bottle fixed to the side of the bed. As in the preceding cases, the wound in the parietes was closed right up to the tube. After a few hours the vomiting had ceased, and within less than 20 hours there was practically a flat abdomen, while the patient was taking milk and manifesting an interest in her dietary. A couple of days later a right basic pneumonia developed, but otherwise the progress to recovery was not seriously interrupted.

It is to be noted that in Case 6. as in the others, no serious effort was made during cæcostomy to cleanse completely the abdomen of free fluid. 'This is in conformity with my view that in post-operative septic peritonitis the patient, generally speaking, dies of the bowel rather than of the peritoneum.

The remarkable recoveries in the foregoing cases appear to me to supply fair proof that intestinal drainage must stand as a principle of surpassing value in the treatment of septic peritonitis. The full recognition of the importance of this principle, and its early application would, I believe, save many lives otherwise inevitably sacrificed. It would, of course, be too much to expect that, as in my present series of post-operative cases, there should always be 100 per cent. of successes. But if this treatment, on an extended scale, should achieve anything like such a high proportion of recoreries-as on theoretical and practical grounds I believe it willthen in intestinal drainage will be found the solution of a great surgical problem.

Perth, Australia.

\section{THE}

SIGNIFICANCE OF PULSUS ALTERNANS AND SOME OTHER FORMS OF UNEVEN PULSE.

By A. E. CARVER, M.D. Cantab., M.R.C.P.Lond.,

HONORARY PHYSICIAN TO OCKENDEN CONVALESCENT HOME AND THE WESTERN AUXILIARY MILITARY HOSPITAL, TORQUAY.

OF recent years it has been taught and generally accepted that the pulsus alternans is a sign of grave omen indicative of impending catastrophe. Notes of a case in which it persisted for several years, however, have been communicated by Dr. C. O. Hawthorne ${ }^{l}$ in a recent paper, and judging from the history of some of the cases reported. below, it seems that its appearance during convalescence from sickness may be regarded, some. times, at all events, with equanimity; from which it is to be inferred that either all the data are not yet to hand for distinguishing, with certainty, whether an alternating pulse is in fact a pulsus alternans, or, the pulsus alternans does not necessarily imply so grave a cardiac disorganisation as has been supposed.

The cases now reported are divided into those who complained of cardiac symptoms and who were, in most instances, found to be the subject of heart disease in its clinical aspects; and others who were recovering from a state of toxæmia and for the most part exhibited neither symptoms nor physical signs of cardiac disablement. The patients were of divers ages and of various occupations and positions in life; and there seems to be no condition common to them excepting that they had all been subject to strain of some kind or another. Those patients in whom the symptoms pointed primarily to a cardiac derangement were of a type that is wont to attain success by personal effort, energetic and active in mind and body, upon whom the wear and tear of a long or worrying life imposes a strain that may not be experienced by a more phlegmatic and less industrious brother; whilst in those who were making recovery from an intoxication the strain was of a temporary nature, incapacitating whilst it lasted, but ceasing with the complete elimination of the exciting cause. Of the former all were males, and three of the four are dead, the surviving case having only recently come under observation. Of the latter two of the five are women, and all are living.

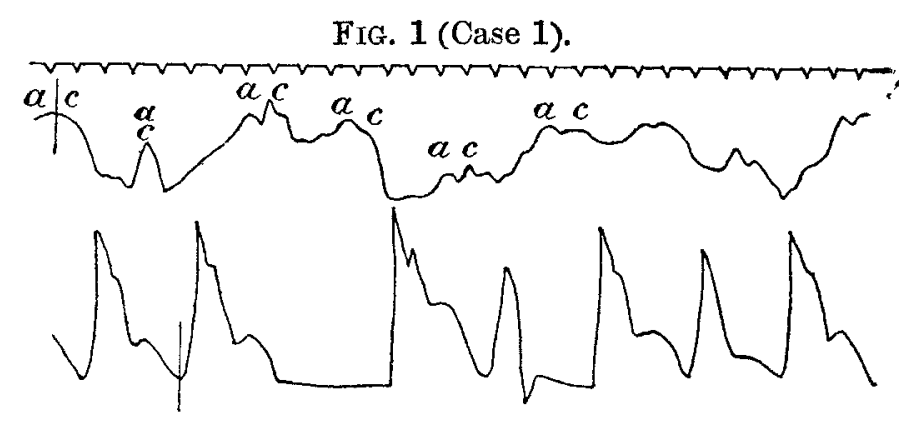

CASE 1.-The patient was an active business man 54 years of age. When he presented himself for advice he complained of breathlessness and præcordial pain; the pulse-rate was perceptibly alternating and murmurs were audible over the mitral and aortic areas. Death ensued 20 months later.

OASE 2.-The patient was a country gentlemen 74 years of age. Whilst on a visit he was seized with angina and subsequently he endured attacks of acute pulmonary cedema,

1 The LANCeT, May 29th, 1915, p. 125. 